\title{
Pressure and Flow Waveform Characteristics of Eight High-Frequency Oscillators
}

\author{
Edward R. Harcourt, BSc ${ }^{1,2}$; Jubal John, $\mathrm{MD}^{3}$; Peter A. Dargaville, MD ${ }^{4,5}$; Emanuela Zannin, \\ $\mathrm{PhD}^{6}$; Peter G. Davis, $\mathrm{MD}^{3,4,7}$; David G. Tingay, $\mathrm{PhD}^{1,3,4,7}$
}

$\mathrm{H}$ igh-frequency oscillators deliver complex airway pressure and gas flow waveforms, at least at the airway opening, to achieve gas exchange (1-3). Early oscillators used different mechanisms to generate oscillations, and each demonstrated unique in vitro pressure and flow characteristics $(1,2)$. Of these early devices, only the Sensormedics 3100A (SM3100, CareFusion, San Diego, CA) and Babylog 8000+ (BL8000, Drägerwerk, Lübeck, Germany) are still commercially available. The performance characteristics of these oscillators (1,2, 4-6), and how clinicians apply high-frequency oscillatory ventilation (HFOV) with them, are different. These differences have been attributed to the method of waveform generation and led to oscillators being classified according to their mechanism of waveform generation $(5,7)$. Since then a new Sensormedics 3100 device (3100B) and five other new oscillators have become commercially available. The manufacturer's described mechanism of generating oscillations differs between machines. The waveform characteristics of these new devices are not known and traditional methods of
${ }^{1}$ Neonatology, Royal Children's Hospital, Parkville, VIC, Australia. ${ }^{2}$ Clinical Technology Service, Royal Children's Hospital, Parkville, VIC, Australia.

${ }^{3}$ Neonatal Research, Royal Women's Hospital, Parkville, VIC, Australia.

${ }^{4}$ Neonatal Research, Murdoch Children's Research Institute, Parkville, VIC, Australia.

${ }^{5}$ Department of Paediatrics, Royal Hobart Hospital, Hobart, TAS, Australia. ${ }^{6}$ Dipartimento di Elettronica, Informazione e Bioingegneria, Politecnico di Milano University, Milano, Italy.

${ }^{7}$ Departments Paediatrics and Obstetrics and Gynaecology, University of Melbourne, Melbourne, VIC, Australia.

Supplemental digital content is available for this article. Direct URL citations appear in the printed text and are provided in the HTML and PDF versions of this article on the journal's website.

Dr. Tingay is supported by a National Health and Medical Research Council Clinical Research Fellowship (Grant ID 491286) and the Victorian Govern-ment Operational Infrastructure Support Program. The remaining authors have disclosed that they do not have any potential conflicts of interest.

For information regarding this article, E-mail: david.tingay@rch.org.au 
characterizing HFOV based on the oscillation generation may no longer be relevant.

The aim of this benchtop study was to describe the pressure and flow waveform characteristics of eight commercially available oscillators in an in vitro setting.

\section{METHODS}

The following oscillators were studied using the manufacturer's recommended circuit without humidification: SM3100A and SM3100B, Fabian (Acutronic, Hirzel, Switzerland), Sophie (Stephan GmbH Medizintechnik, Gackenbach, Germany), SLE5000 (SLE UK, Croyden, United Kingdom), Leonie+ (Heinen+Löwenstein, Bad Ems, Germany), and Dräger's BL8000 and the new Babylog VN500.

\section{Experimental Setup}

A 15-cm long 3.5-mm internal diameter endotracheal tube (Mallinckrodt, Rowville, Victoria, Australia) was connected to an infant test lung with a residual lung volume of $70 \mathrm{~mL}$ (model 560li; MI Instruments, Grand Rapids, MI), creating a leak-free circuit. Test lung compliance was set to $1 \mathrm{~mL} / \mathrm{cm} \mathrm{H}_{2} \mathrm{O}$ (lowest possible setting). Flow was measured at the airway opening $\left(V_{\mathrm{AO}}^{\prime}\right)$ using a hot wire anemometer sampling at $200 \mathrm{~Hz}$ (Florian Respiratory Monitor, Acutronic) (8). Airway pressure was measured at 1,000 $\mathrm{Hz}$ using calibrated pressure transducers (SC-24, Scireq, Montreal, Canada) located at the proximal end of the circuit (pressure in the ventilator $\left[P_{\mathrm{VENT}}\right]$ ), the airway opening (pressure at the airway opening $\left[P_{\mathrm{AO}}\right]$ ), and within the test lung (pressure in the "trachea" of the test lung $\left[P_{\mathrm{TRACH}}\right]$ ).

\section{Oscillation Strategy}

Each oscillator, in standard HFOV mode, was used to ventilate the test lung at mean airway pressure $\left(P_{\mathrm{AW}}\right)$ of 10 and $20 \mathrm{~cm} \mathrm{H}_{2} \mathrm{O}$ and frequencies of 5,10 , and $15 \mathrm{~Hz}$. Inspiratory to expiratory time ratio (I:E) was set at $1: 2$, except on the SLE5000 which operates with a fixed 1:1 I:E ratio. To allow comparison, SM3100A and SM3100B were also tested with a 1:1 I:E ratio (3). Pressure amplitude $(\Delta P)$ was $30 \mathrm{~cm} \mathrm{H}_{2} \mathrm{O}$ in oscillators that used absolute units. For the ventilators that set $\Delta P$ as a percentage (Sophie and BL8000), the \%Max was titrated to deliver $30 \mathrm{~cm} \mathrm{H}_{2} \mathrm{O}$ trough-to-peak measurement of the $P_{\text {VENT }}$ waveform.

\section{Data Acquisition and Analysis}

Analog signals were acquired over 600 consecutive oscillatory cycles and digitalized using LabChart (V7.2.1, AD Instruments, Sydney, Australia). $P_{\mathrm{VENT}}, P_{\mathrm{AO}}, P_{\mathrm{TRACH}}$, and $V_{\mathrm{AO}}^{\prime}$ waveforms were plotted and tidal volume $\left(V_{\mathrm{T}}\right)$ calculated. The waveforms were characterized using the following criteria: square or sine wave and presence of unique incisurae. Peak, trough, and amplitude of each waveform were determined. Power spectral density (PSD) analysis was performed on the $P_{\mathrm{AO}}$ waveform using Welch's periodogram method in Matlab (v7.1 SP3, Natick, MA) with a frequency resolution of $0.5 \mathrm{~Hz}$. This process allows for oscillator waveform to be separated into its different harmonics, and thus, analysis (as a graphical representation) of the nonfundamental frequencies that contribute to the high-frequency waveform at $10 \mathrm{~Hz}$ performed. The power, or strength, of each nonfundamental frequency is graphically expressed relative to the power of the fundamental frequency (represented with a value of 1) (2). This separation can also be expressed as the ratio of the signal power of fundamental frequencies to the power of the nonfundamental frequency (FTN) using fast Fourier transformation. A lower FTN represents a waveform with denser harmonic composition, which theoretically leads to less waveform distortion and better energy transmission in the respiratory tree.

\section{RESULTS}

There was no difference in the results at $P_{\mathrm{AW}} 10$ and $20 \mathrm{~cm} \mathrm{H}_{2} \mathrm{O}$, and the $10 \mathrm{~cm} \mathrm{H}_{2} \mathrm{O}$ data are presented.

\section{Waveforms}

The $P_{\mathrm{AO}}$ and $V_{\mathrm{AO}}^{\prime}$ of each oscillator are shown in Figure 1. Oscillators exhibited either a square or sine wave pattern with varying harmonic components. The Fabian, Leonie+, and BL8000 $P_{\mathrm{AO}}$ and flow profiles resembled sine waves. The VN500 pressure waveform was a pure sine wave with a sheer expiratory slope. The SM3100B $P_{\mathrm{AO}}$ and $V_{\mathrm{AO}}^{\prime}$ waveforms at $1: 2$ $\mathrm{I}: \mathrm{E}$ ratio differed from the essentially square waves at $1: 1 \mathrm{I}$ :E ratio and the SM3100A at both I:E settings. At 1:2 ratio, the SM3100B waveforms exhibited an initial inspiratory pulse and incisurae, then stepwise reductions in pressure and flow preceding peaks. The SLE5000 exhibited a square $P_{\mathrm{AO}}$ that was comparable to SM3100A and B at 1:1 ratios. The Sophie generated sine pressure and flow waves, but unlike the other sine wave devices, the pressure waveform exhibiting incisurae on the inflation slope similar to the square wave devices. The BL8000 $P_{\mathrm{AO}}$ waveform had a small notch in the inspiratory slope without a distinct incisurae. Across all devices, the general shape of the waveforms did not change throughout the frequency range. In particular, the location of any incisurae was not altered.

Figure 2 shows the $P_{\text {TRACH }}$ waveform for each device. Compared with the $P_{\mathrm{AO}}$ waveform, all devices generated a sine wave pattern within the test lung. There was evidence of a more irregular wave in the square waved devices (SM3100A and B and SLE5000) but any incisurae had been attenuated.

\section{Delivered Pressure and Flow at Experimental Settings}

The operational range of the ventilators varied at comparable settings, as shown in Table $1(10 \mathrm{~Hz})$ and Supplemental Digital Tables $1(5 \mathrm{~Hz})$ and $2(15 \mathrm{~Hz})$ (Supplemental Digital Content 1, http://links.lww.com/PCC/A96). All oscillators, except the BL8000, generated a $\Delta P_{\text {VENT }}$ above $28.9 \mathrm{~cm} \mathrm{H}_{2} \mathrm{O}$ and $V_{\mathrm{T}}$ greater than $6 \mathrm{~mL}$ at frequencies above $10 \mathrm{~Hz}$. At the settings tested, all but the BL8000 and SM3100A (1:2 ratio) were able to achieve sub- or atmospheric airway pressures during expiration. Minimum flow and pressure became more negative as frequency increased in the SLE5000. Delivered $V_{\mathrm{T}}$ was generally similar between devices at comparable I:E ratios, except for 


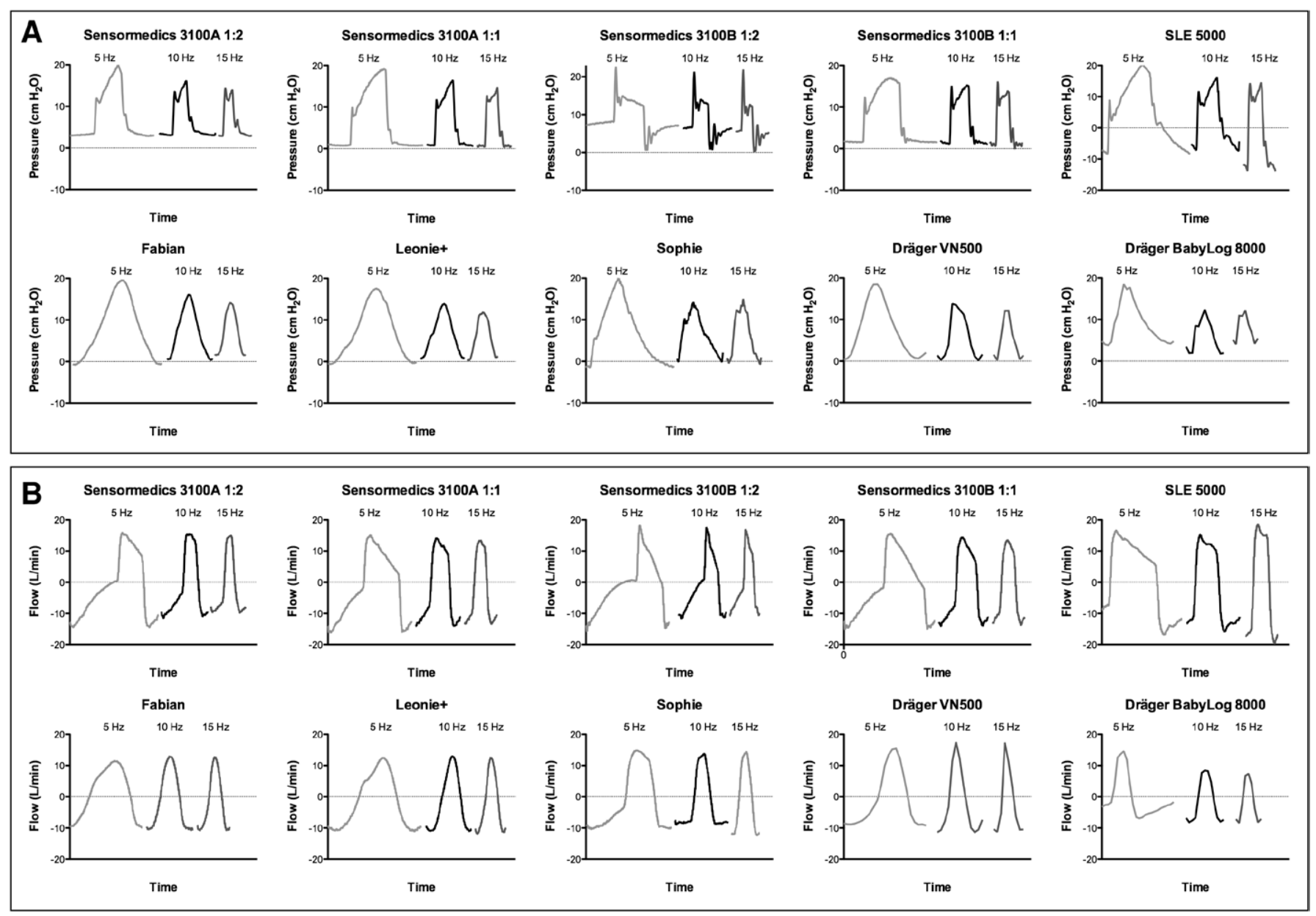

Figure 1. Pressure (A) and flow (B) waveforms at the airway opening during a single oscillation at $5 \mathrm{~Hz}$ (light gray), $10 \mathrm{~Hz}$ (black), and $15 \mathrm{~Hz}$ (dark gray) for the eight high-frequency oscillators evaluated. Devices defined as having a square-shaped pressure waveform are in the first row and the sine wave devices in the second row. All recordings at an inspiratory:expiratory ratio of 1:2 except the SLE5000 (1:1) and SM3100B (1:2 and 1:1).
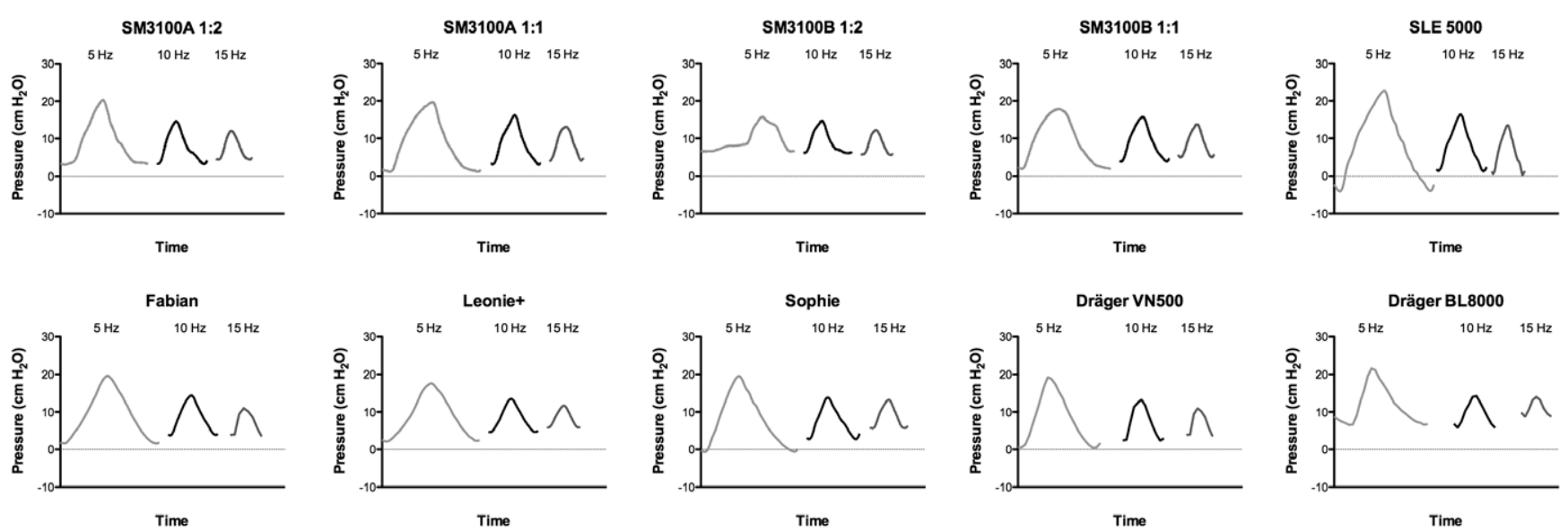

Figure 2. Pressure waveform within the test lung (pressure in the "trachea" of the test lung during a single oscillation at $5 \mathrm{~Hz}$ (light gray), $10 \mathrm{~Hz}$ (black), and $15 \mathrm{~Hz}$ (dark gray) for the eight high-frequency oscillators evaluated. All devices generated a sine wave within the lung. All recordings at an inspiratory:expiratory ratio of 1:2 except the SLE5000 (1:1) and SM3100B (1:2 and 1:1).

the BL8000. Different frequencies did not alter $\Delta P_{\mathrm{VENT}}, \Delta P_{\mathrm{AO}}$, and $V_{\mathrm{AO}}^{\prime}$, but, as expected, delivered $V_{\mathrm{T}}$ and $\Delta P_{\text {TRACH }}$ decreased in all devices with increasing frequency.

\section{Power Spectral Density}

The PSD of the $P_{\mathrm{AO}}$ waveform for each ventilator is shown in Figure 3 and the FTN in Figure 4. The spectral density 


\section{TABLE 1. Operational Pressure and Flow Range and Principles of Operations of Eight High-Frequency Oscillatory Ventilators at 10-Hz Frequency}

\begin{tabular}{|c|c|c|c|}
\hline Ventilator & Principle of Operation & $\begin{array}{c}\text { Inspiratory: Expiratory } \\
\text { Ratio }\end{array}$ & $\begin{array}{l}\text { Pressure in the } \\
\text { Ventilator }\left(\mathrm{cm}_{2} \mathrm{O}\right)\end{array}$ \\
\hline Sensormedics 3100A & Electromagnetic flow generator & $1: 2$ & 30.5 \\
\hline Sensormedics 3100A & As above & $1: 1$ & 30.9 \\
\hline Sensormedics 3100B & Electromagnetic flow generator & $1: 2$ & 35.6 \\
\hline Sensormedics 3100B & As above & $1: 1$ & 32.2 \\
\hline SLE5000 & Bidirectional jets & $1: 1$ & 35.9 \\
\hline Fabian & Voice coil flow generator & $1: 2$ & 32.1 \\
\hline Leonie+ & Membrane-integrated diaphragms & $1: 2$ & 28.9 \\
\hline Sophie ${ }^{a}$ & Valve oscillator with active expiration & $1: 2$ & 30.0 \\
\hline VN500 & Expiratory valve with venturi-assisted expiration & $1: 2$ & 33.5 \\
\hline $\mathrm{BL} 8000^{\mathrm{b}}$ & Expiratory valve with venturi-assisted expiration & $1: 2$ & 16.3 \\
\hline
\end{tabular}

$P_{\mathrm{AO}}=$ pressure at the airway opening, $V_{\mathrm{AO}}^{\prime}=$ flow at the airway opening.

a\%Max $\Delta P$ of $40 \%$ was required to achieve $30 \mathrm{~cm} \mathrm{H}_{2} \mathrm{O}$ pressure in the ventilator $\left(\Delta P_{\text {VENT }}\right)$ for the Sophie oscillator.

${ }^{\mathrm{b}} \mathrm{BL} 8000$ was unable to achieve $30 \mathrm{~cm} \mathrm{H}_{2} \mathrm{O} \Delta P_{\mathrm{VENT}}$ at $100 \% \mathrm{Max} \Delta P$; the value shown is the highest $\Delta P$ the ventilator could produce at these settings.

$5 \mathrm{~Hz}$ and $15 \mathrm{~Hz}$ data available in Supplemental Digital Tables 1 and 2 (Supplemental Digital Content 1, http://links.lww.com/PCC/A96).

Recordings made at settings of mean airway pressure $10 \mathrm{~cm} \mathrm{H}_{2} \mathrm{O}$, frequency $10 \mathrm{~Hz}$, pressure amplitude $(\Delta P) 30 \mathrm{~cm} \mathrm{H}_{2} \mathrm{O}$, compliance $1.0 \mathrm{~mL} / \mathrm{cm} \mathrm{H} \mathrm{H}_{2} \mathrm{O}$, and inspiratory:expiratory ratio as specified via a $3.5-\mathrm{mm}$ endotracheal tube.

for the square wave ventilators conformed to known square wave characteristics with peaks at odd multiples for 1:1 I:E ratios and consecutive peaks for 1:2 ratios. Oscillators generating square waves (SM3100A and B, SLE5000) and/or a distinct inspiratory slope incisurae (Sophie) generated waveforms with a higher number of nonfundamental frequency components, and thus the most complex frequencies with potentially greatest transmissive power. Generally, as the fundamental frequency becomes more dominant, with less harmonic (nonfundamental) components, the FTN became more positive. At $5 \mathrm{~Hz}$, the $\mathrm{SM} 3100 \mathrm{~A}$ and $\mathrm{B}$ at 1:2 I:E ratio generated a pressure waveform composed of some nonfundamental frequencies with a higher signal power than the fundamental frequency $(5 \mathrm{~Hz})$. All other ventilators had FTN greater than 1.0 across the range of frequencies except the VN500. Overall, as the frequency increased, the FTN changed in a nonuniform manner. The VN500 was the only sine wave device to show a large change in FTN with increasing frequency. At $15 \mathrm{~Hz}$, the FTN had decreased to 0.71 , or $60 \%$ from the $5-\mathrm{Hz}$ value, as demonstrated by the marked change in PSD of the $P_{\mathrm{AO}}$ waveform.

\section{DISCUSSION}

This is the first study to describe the waveform characteristics of the current high-frequency oscillators. We found differences in the shape and complexity of the generated pressure and flow waveforms. Traditionally, high-frequency oscillators have been classified according to the mechanism used to generate high-frequency waveforms (e.g., oscillator and flow-interrupter) (7). Our study suggests that the complexity of engineering design in the modern oscillators, and resultant waveforms, makes this approach to nomenclature obsolete. We propose that using the characteristics of the waveforms along with frequency composition provides a more useful method for classifying modern high-frequency oscillators.

We demonstrated marked differences at the airway opening in the generated waveforms and the absolute $\Delta P_{\mathrm{VENT}} \Delta P_{\mathrm{AO}}$, and $V_{\mathrm{AO}}^{\prime}$ values. Unlike conventional ventilation, HFOV is reliant on the waveforms generated to achieve gas exchange at small tidal volumes $(1,2)$. The SM3100A and BL8000 are known to produce very different waveforms, which lead to clinically important differences in performance $(4-6,9)$. Unlike the SM3100 oscillators, modern ventilators are hybrid devices, offering conventional and high-frequency modes. Only the SLE5000 produced a waveform similar to the SM3100 oscillators. Whether delivery of a sine wave influences clinical performance or effectiveness cannot be determined from our study. Pressure waveforms are known to attenuate to sine waves at the alveolar level in the SM3100A $(1,10)$, and this was apparent in all the devices we evaluated. $V_{\mathrm{T}}$ was similar at comparable I:E ratios across all oscillators in our study, with the exception of SM3100 A and B (3) and BL8000 $(4,6)$ where differences in generated $V_{\mathrm{T}}$ have been described previously. Especially at lower frequencies, the $V_{\mathrm{T}}$ at the airway opening was above the dead space of the respiratory system, irrespective of waveform type. This observation is consistent with previous benchtop and human studies (11-14), the difference being that some of the new oscillators now offer high-frequency tidal volume targeting modalities. The relationship between HFOV waveforms, 


\begin{tabular}{|c|c|c|c|c|c|}
\hline $\begin{array}{l}\text { Peak } P_{A O} \\
\left(\mathrm{~cm} \mathrm{H}_{2} \mathrm{O}\right)\end{array}$ & $\begin{array}{c}\text { Trough } P_{A O} \\
\left(\mathrm{~cm} \mathrm{H}_{2} \mathrm{O}\right)\end{array}$ & $\begin{array}{l}\text { Pressure in the } \\
\text { "Trachea" of the Test } \\
\text { Lung }\left(\mathrm{cm} \mathrm{H}_{2} \mathrm{O}\right)\end{array}$ & $\begin{array}{c}\text { Peak } V_{\mathrm{AO}}^{\prime} \\
(\mathrm{L} / \mathrm{min})\end{array}$ & $\underset{(\mathrm{L} / \mathrm{min})}{\operatorname{Trough} V_{\mathrm{AO}}^{\prime}}$ & $\begin{array}{c}\text { Tidal } \\
\text { Volume }(\mathrm{mL})\end{array}$ \\
\hline 16.3 & 3.0 & 11.5 & 15.6 & -11.2 & 6.9 \\
\hline 16.5 & 0.7 & 13.2 & 14.0 & -14.1 & 7.8 \\
\hline 21.7 & 0.3 & 8.6 & 16.4 & -10.4 & 4.6 \\
\hline 17.0 & 1.1 & 11.9 & 14.4 & -13.9 & 8.6 \\
\hline 16.2 & -7.2 & 15.2 & 16.3 & -15.8 & 8.3 \\
\hline 15.6 & 0.3 & 9.9 & 12.8 & -10.4 & 6.3 \\
\hline 13.9 & 0.8 & 8.8 & 12.8 & -11.0 & 6.0 \\
\hline 14.3 & -0.3 & 9.6 & 13.7 & -8.9 & 6.6 \\
\hline 13.1 & 0.3 & 10.9 & 17.3 & -11.4 & 6.6 \\
\hline 10.7 & 2.2 & 8.5 & 8.5 & -8.0 & 4.7 \\
\hline
\end{tabular}

generated pressure, and effectiveness in vivo warrants further investigation.

In this study, we attempted to characterize the waveforms in the frequency domain (2). We contend that this is a relatively simple method of describing a waveform's composition, although FTN is not without limitations. The FTN is analogous to the signal-to-noise ratio used in engineering to compare the strength of a desired signal power to the power of background noise. In this context, however, the frequency composition is secondary to the function of the waveform and any analysis using fast Fourier transformation must be careful not to assume "desired" waves and "unwanted" noise. The ratio is used only as a descriptive tool, and until further studies focused on waveform nature and composition are undertaken, these types of analyses should not be used to assume a ventilator's performance or effectiveness. Despite this, it is interesting that the VN500 demonstrated a marked change in the FTN at a frequency of $15 \mathrm{~Hz}$. Recently, the VN500 was found to have a frequency-dependent limitation in oscillatory performance at frequencies above $10 \mathrm{~Hz}(10)$. This illustrates the importance of clinicians understanding the type of oscillator they are using and differences in application with other devices.

This benchtop study was unable to determine whether the marked differences in delivered waveforms are likely to translate into clinical differences in oscillatory performance or lung protection. HFOV has long been advocated as a 1 ung-protective mode, especially when applied with a high lung volume strategy (15). The role of oscillator design in lung protection remains unclear. The SM3100A was the sole device used in a trial that demonstrated better lung protection in preterm infants compared to conventional ventilation when HFOV was used with a high lung volume strategy (15). Conversely, the United Kingdom Oscillation Study trial suggested the least benefit from the SM3100A compared to other oscillators (16), although it has been argued that this trial did not use a true high lung volume strategy (17). More recently, individual patient meta-analysis has failed to show a difference in oscillator type on lung injury (18). Despite this uncertainty, these studies highlight the importance of applying HFOV optimally, irrespective of device.

All devices achieved similar pressure waveforms at the $P_{\text {TRACH }}$ level, demonstrating significant attenuation of the oscillatory wave as it enters the upper respiratory tree. We did not evaluate the flow patterns within the test lung, in part, due to the limitation of the test lung used and also as clinicians are only able to see flow at the airway opening. Interestingly, subtle irregularities in the sine wave pattern were evident in the square wave devices. This finding is intriguing and warrants further evaluation in more complex in vivo models to determine whether these differences translate to differences at an alveolar level.

\section{Limitations}

This was a benchtop test using a test lung with known performance limitations. In particular, the mechanical test lung does not exhibit the complex viscoelastic properties of the diseased human lung. This explains the similarity in results at $P_{\mathrm{AW}} 10$ and $20 \mathrm{~cm} \mathrm{H}_{2} \mathrm{O}$. The circuits tested were those recommended by the manufacturers, and not the same across devices. Thus, 
$5 \mathrm{~Hz}$
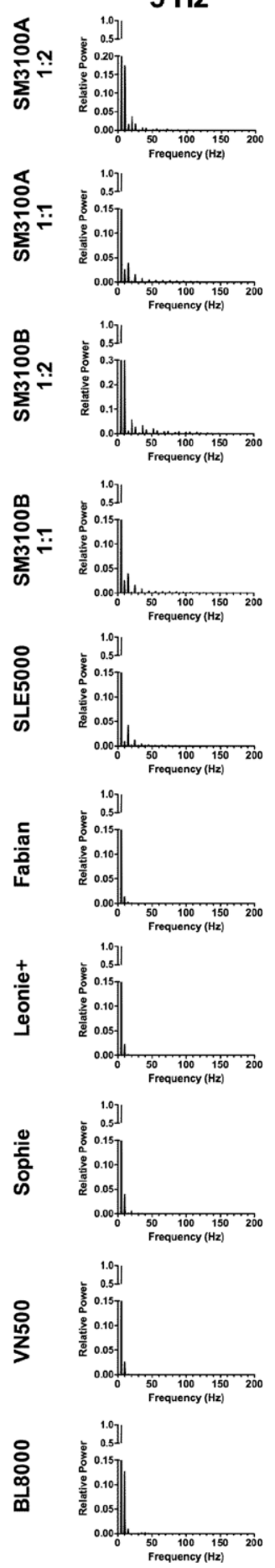

Figure 3. Power spectral density of ventilator pressure at the airway opening waveform at $5 \mathrm{~Hz}(\mathbf{A}), 10 \mathrm{~Hz}(\mathbf{B})$, and $15 \mathrm{~Hz}(\mathbf{C})$. Subsequent peaks represent power of $\boldsymbol{x}$-axis frequency relative to fundamental, or set, frequency.

the flow waveform characteristics generated within the oscillators may vary. The Sophie and BL8000 set the $\Delta P$ as percentage of maximum available power. The maximum available power changes with many factors including respiratory system compliance, $P_{\mathrm{AW}}$, and frequency. Waveform characteristics may thus change without altering oscillator settings.

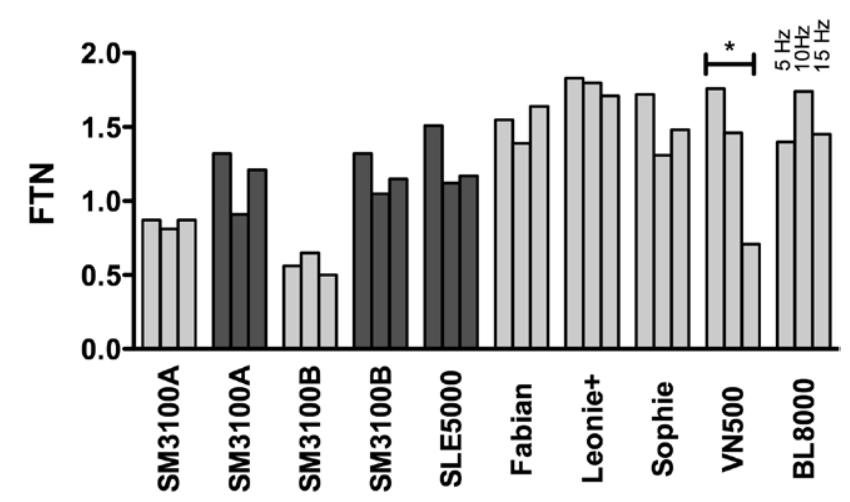

Figure 4. Ratio of the signal power of fundamental frequency to the nonfundamental frequencies (FTN) values at 5,10 , and $15 \mathrm{~Hz}$ frequencies for eight high-frequency oscillatory ventilators. Light gray-filled bars represent data at an inspiratory:expiratory ratio of 1:2 and dark gray bars 1:1. *VN500 demonstrated the greatest change in FTN with increasing frequency.

\section{CONCLUSIONS}

This study has shown that the waveforms generated by modern oscillators differ by shape, frequency characteristics, and resultant amplitudes at comparable settings. Clinicians should be aware of these differences, which may lead to variations in the effectiveness of oscillation between these devices. Further clinical studies are required to identify the in vivo implications of differences in high-frequency waveforms.

\section{REFERENCES}

1. Thome U, Pohlandt F: Effect of the TI/TE ratio on mean intratracheal pressure in high-frequency oscillatory ventilation. J Appl Physiol (1985) 1998; 84:1520-1527

2. Pillow JJ, Wilkinson $\mathrm{MH}, \mathrm{Neil} \mathrm{HL}$, et al: In vitro performance characteristics of high-frequency oscillatory ventilators. Am J Respir Crit Care Med 2001; 164:1019-1024

3. Custer JW, Ahmed A, Kaczka DW, et al: In vitro performance comparison of the Sensormedics $3100 \mathrm{~A}$ and $\mathrm{B}$ high-frequency oscillatory ventilators. Pediatr Crit Care Med 2011; 12:e176-e180

4. Hatcher $\mathrm{D}$, Watanabe $\mathrm{H}$, Ashbury $\mathrm{T}$, et al: Mechanical performance of clinically available, neonatal, high-frequency, oscillatory-type ventilators. Crit Care Med 1998; 26:1081-1088

5. Fredberg JJ, Glass GM, Boynton BR, et al: Factors influencing mechanical performance of neonatal high-frequency ventilators. J Appl Physiol (1985) 1987; 62:2485-2490

6. Jouvet $P$, Hubert $P$, Isabey $D$, et al: Assessment of high-frequency neonatal ventilator performances. Intensive Care Med 1997; 23:208-213

7. Lampland AL, Mammel MC: High-frequency ventilation. In: Assisted Ventilation of the Neonate. Fourth Edition. Goldsmith JP, Karotkin E (Eds). St Louis, Elsevier Saunders, 2010, pp 200-219

8. Scalfaro P, Pillow JJ, Sly PD, et al: Reliable tidal volume estimates at the airway opening with an infant monitor during high-frequency oscillatory ventilation. Crit Care Med 2001; 29:1925-1930

9. Wheeler KI, Schmölzer GM, Morley CJ, et al: High-frequency ventilation with the Drager Babylog 8000plus: Measuring the delivered frequency. Acta Paediatr 2011; 100:67-70

10. Johns JH, Harcourt E, Davis P, et al: Dräger VN500's oscillatory performance has a frequency-dependent threshold. J Paediatr Child Health 2014; 50:27-31

11. Dimitriou G, Greenough A, Kavvadia V, et al: Volume delivery during high frequency oscillation. Arch Dis Child Fetal Neonatal Ed 1998; 78:F148-F150 
12. Pillow JJ: High-frequency oscillatory ventilation: Mechanisms of gas exchange and lung mechanics. Crit Care Med 2005; 33:S135-S141

13. Tingay DG, Mills JF, Morley CJ, et al: Indicators of optimal lung volume during high-frequency oscillatory ventilation in infants. Crit Care Med 2013; 41:232-239

14. Weinmann GG, Mitzner W, Permutt S: Physiological dead space during high-frequency ventilation in dogs. J App/ Physiol Respir Environ Exerc Physiol 1984; 57:881-887

15. Courtney SE, Durand DJ, Asselin JM, et al; Neonatal Ventilation Study Group: High-frequency oscillatory ventilation versus conventional mechanical ventilation for very-low-birth-weight infants. N Engl J Med 2002; 347:643-652
16. Johnson AH, Peacock JL, Greenough A, et al; United Kingdom Oscillation Study Group: High-frequency oscillatory ventilation for the prevention of chronic lung disease of prematurity. N Engl J Med 2002; 347:633-642

17. van Kaam $A H$, Rimensberger PC: Lung-protective ventilation strategies in neonatology: What do we know-What do we need to know? Crit Care Med 2007; 35:925-931

18. Cools F, Askie LM, Offringa M, et al; PreVILIG collaboration: Elective high-frequency oscillatory versus conventional ventilation in preterm infants: A systematic review and meta-analysis of individual patients' data. Lancet 2010; 375:2082-2091 